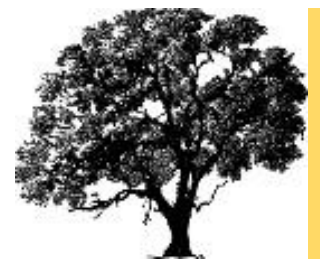

Eurasian Journal of Forest Science

2021 9(3): 259-271

http://dergipark.org.tr/en/pub/ejejfs

\title{
Pollen analysis of honeys from Yığılca region (Düzce), Turkey
}

\author{
Elif Ayşe YILDIRIM ${ }^{1{ }^{*}}$ (D), Nurgül KARLIOĞLU KILIÇ ${ }^{1}$ (D), Neval Güneş ÖZKAN² (D) \\ 1,* Istanbul University-Cerrahpaşa, Faculty of Forestry, 34473, Istanbul, Turkey \\ 1,* Duzce University, Faculty of Forestry, 81620. Duzce, Turkey
}

\begin{abstract}
Corresponding Author: elifyildirim755@gmail.com

The aim of this study is to determine the plant species preferred by Apis mellifera L., which is a valuable genotype in terms of vitality and high honey production. For this purpose, seven honey samples from Yığılca region in Düzce (Turkey) were examined for pollen analysis. As a result of the pollen analysis on these samples, it was determined that Yı̆̆llca local honeybee use pollen grains of 43 different taxa from 26 families. Six samples of honey were unifloral (> $45 \%$ pollen of one taxon in sample) with four being unifloral for Rhododendron ponticum L. and two for Castanea sativa Mill. One sample of honey was classified as multifloral with the percentages of secondary and rare pollen groups. The honeys had contained 14 to 30 different types of pollen. The average number of pollen types per honey was 21. The dominant groups of pollen grains were determined as Rhododendron ponticum L. in 4 samples and Castanea sativa Mill. in 2 samples. Secondary pollen components were from 3 taxa: Fagus orientalis Lipsky, Rhododendron ponticum L. and Quercus L. sp. Four pollen types occurred as 'minor' types and pollen grains of 37 taxa were in rare quantities. Asteraceae, Fabaceae, Fagaceae, Lamiaceae and Rosaceae were the most represented families in honey samples.
\end{abstract}

Keywords: Castanea sativa, honeybee, honey, pollen, pollen analysis, Rhododendron ponticum, Yı̆̆ılca region.

\section{Introduction}

Melissopalynology (pollen analysis in honey), which is one of the sub-branches of palynology, provides information about the floristic origin of honey where it is produced (Sawyer 1981). Also, this analysis determines the quality and classification of honey samples (Özler 2015). The identification of unifloral honeys is important for commercial and scientific interest (Silici and Gökçeoğlu 2007). Honey obtained from a single flower is defined as "monoflora" and honey consisting of mixed flowers is defined as "polyflora" (Sorkun 2008). The first study about the pollen analysis of honey in Turkey was performed by Qustrani in 1976 (Erdoğan et al. 2006). Many studies about pollen spectrum of honeys were carried out in the different regions of Turkey (Sorkun and İnceoğlu 1984, Sorkun and Doğan 1995, Doğan and Sorkun 2001, Kaya et al. 2005, Erdoğan et al. 2006, Silici and Gökçeoğlu 2007, Sorkun 2008, Özler 2015, 2018, Ş1k et al. 2017, Altay et al. 2018).

The existence of the 'Yı ğılca bee' genotype, which is unique to this region, is the most important reason for choosing the "Yığılca Balköy Honey Production Forest" as the study area. Considering the natural flora of the region, rhododendron (Rhododendron ponticum L.), chestnut (Castanea sativa Mill.), clover (Trifolium L. sp.), sage (Salvia L. sp.) are important and valuable plant taxa preferred by bees in honey production. The high and valuable honey production power of the Yiğılca bee has brought beekeeping and honey production activities in this region, and thus beekeeping has been an important economic 
income for the local people. Gösterit et al. (2012) compared Yığılca bee with Anatolian and Caucasian bees and they determined that Yığllca bee is a valuable bee genotype in terms of vitality and high honey production, and it stores more honey than other genotypes. Kekeçoğlu (2007) indicated that the Yı̆̆ılca bee ecotype has characteristics that are exceedingly above the Turkey average in terms of tongue and wing length, which are extremely determining morphological structures for honey bees. Kambur et al. (2015) examined the chemical structure and pollen content of honey samples taken from the vicinity of Yığılca district of Düzce province during the 2015 honey harvest season, and 10 honey samples were analyzed and 3 samples were determined as monofloral and 7 samples were determined as multifloral honey in this study. The dominant pollen families of monofloral honeys were Ericaceae (Rhododendron ponticum L.) and Fagaceae. The secondary pollen families of multifloral honeys were determined as Fabaceae, Fagaceae, Poaceae, Apiaceae and Asteraceae. Kambur et al. (2015) identified pollen grains of honey samples according to the family level. However, the pollen spectrum of the honeys taken from the same research area was revealed in the taxon level in this present study. Thus, this study aims to find out the floral composition of Yığılca honey samples by identification and characterization of pollen content in a detailed microscopic analysis.

\section{Material and Method}

\subsection{Geographical status of the study area}

Y1ğılca Balköy Honey Production Forest, chosen as the study area, is located between 40 56'24" -

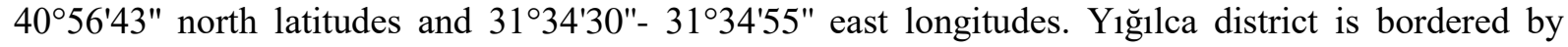
Zonguldak province in the north, Bolu province in the east and south, Düzce province in the west, Akçakoca district in the northwest and Kaynaşlı district in the southwest (Figure 1). It has a generally mountainous topography. The highest point of the study area is 1100 meters and the lowest point is 700 meters. The area is located within the boundaries of Kurtkayas1 Forestry Operation Directorate, which is affiliated to Yığılca Forest Management Directorate, and is located around Çukurören and Yoğunpelit villages (Y1lmaz et al. 2017).

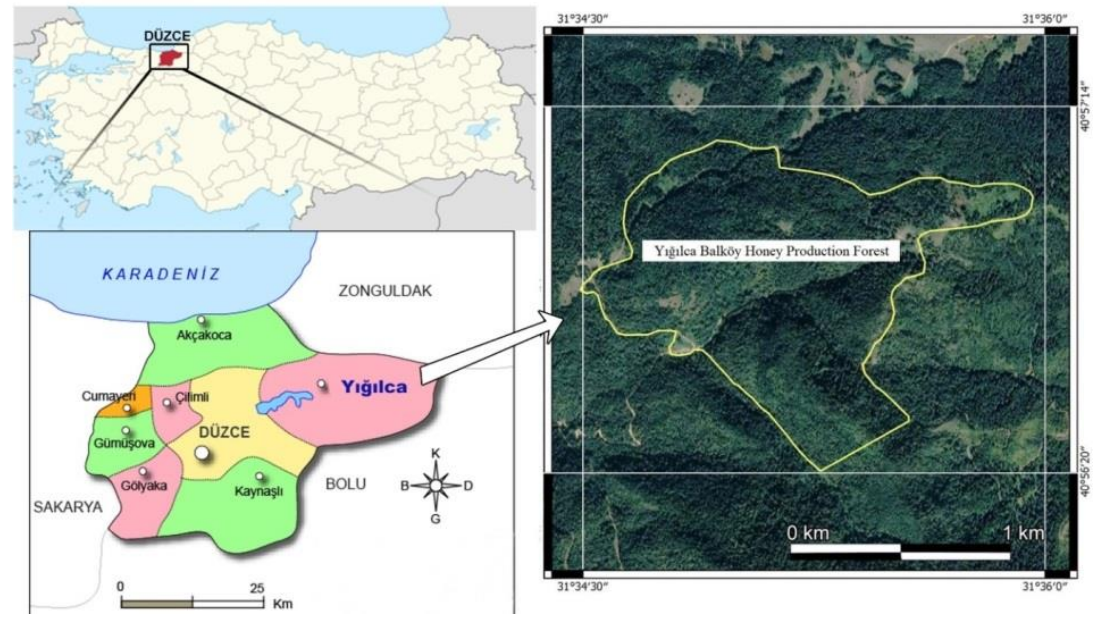

Figure 1: Location map of the study area (Google Maps).

\subsection{Flora of Yığılca Balköy Honey Production Forest}

The study area is located between the Euro-Siberian and the Mediterranean flora region. It is situated in A3 square according to the grid system of Davis (1965). A total of 159 plants were collected during the field studies between 2019-2020. As a result of the plant identification, a total of 137 taxa belonging to 46 families and 104 genera were determined. The distribution of plant taxa in the study area according to phytogeographical regions was determined as $41,61 \%$ in Euro-Siberian, $0,73 \%$ in Irano-Turanian, 
$3,65 \%$ in the Mediterranean, and 54,01\% in those with a wide distribution or whose phytogeographical region is unknown. The family with the most genera is Asteraceae with 15 genera and the family with the most species is Asteraceae with 21 species. The largest genus is Cirsium with 5 taxa. Important woody species of this forest were determined as Acer campestre L., Alnus glutinosa (L.) Geartner subsp. glutinosa, Carpinus betulus L., Castanea sativa Miller, Cornus sanguinea L. subsp. australis (C.A.Mey.) Jáv., Corylus avellana L. var. avellana, Crataegus monogyna Jacq. var. monogyna, Erica arborea L., Euonymus latifolius (L.) Mill. subsp. latifolius, Fagus orientalis Lipsky, Ilex colchica Pojark., Juglans regia L., Ligustrum vulgare L., Mespilus germanica L., Phillyrea latifolia L., Pinus sylvestris L., Prunus spinosa L., Pyracantha coccinea Roemer., Pyrus elaeagnifolia Pall. subsp. elaeagnifolia, Quercus cerris L. var. cerris, Quercus frainetto Ten, Quercus pubescens Willd., Rhododendron ponticum L., Rosa canina L., Rubus L. sp., Staphylea pinnata L. and Tilia tomentosa Moench. (Yıldırım et al. 2020).

\subsection{Honey samples and reference slides}

Seven samples of honey produced in the vicinity of Yığılca Balköy Honey Production Forest taken from different producers between May and August 2019 were brought to the Palynology Laboratory of Forestry Faculty of Istanbul University-Cerrahpaşa and pollen analyzes were made on these samples (Table 1).

Table 1: Samples of Yı̆̆

\begin{tabular}{|c|c|c|}
\hline Honey samples & Location & Botanical origin \\
\hline Y01 & Düzce (Yı̆̆ılca) & Rhododendron (Monofloral) \\
\hline Y02 & Düzce (Yı̆̆ılca) & Rhododendron (Monofloral) \\
\hline Y03 & Düzce (Yı̆̆ılca) & Blossom (Multifloral) \\
\hline Y04 & Düzce (Yı̆̆ılca) & Blossom (Multifloral) \\
\hline Y05 & Düzce (Yığılca) & Chestnut (Monofloral) \\
\hline Y06 & Düzce (Yığılca) & Rhododendron (Monofloral) \\
\hline Y07 & Düzce (Y1ğılca) & Blossom (Multifloral) \\
\hline
\end{tabular}

Collected plants in the field work were identified and stored in the herbarium of Düzce University (DUOF). Reference slides of pollen grains from identified plants were made according to the method of Louveaux (1970). Anthers were removed from flower buds of fresh and herbarium plants and the pollen grains were concentrated using a drop of alcohol in the center of a glass slide. The grains were then placed in glycerin jelly on glass slides and sealed with paraffin. These slides were included in the reference slide collection at the Palynology Laboratory of Istanbul University-Cerrahpaşa for later comparison with the pollen types found in the honey samples.

\subsection{Extraction of honey sediment for microscopic analysis}

The preparation of honey samples followed the standardized method of Louveaux (1970) and Sorkun (2008). Ten gram of well homogenized honey was dissolved in $20 \mathrm{ml}$ distilled water. The tubes were kept in a $45{ }^{\circ} \mathrm{C}$ water bath for 10-15 minutes in order to dissolve the honey in water. The solution was centrifuged at $3500 \mathrm{rpm}$ (revolutions per minute) for 10 minutes and the decanted sediment was washed with 5 to $10 \mathrm{ml}$ of distilled water. Upper water phase was discarded and glycerol-gelatin mixture (1:1.5) was added to the tubes. They were transferred to the slides, covered with coverslip and prepared for microscopic analysis. Leica DM750 brand light microscope was used to make pollen analyzes and to take photos of the pollen grains in the slides. Generally $\mathrm{x} 40$ and $\mathrm{x} 100$ objectives were used for the pollen identification. Each preparation was scanned starting from the upper left corner and different pollen types found in the slides were identified on the basis of family, genus and species with the aid of prepared 
reference collection and with the use of microphotographs and reference pollen atlases from the literature (Woodhouse 1935, Erdtman 1952, Erdtman 1957, Faegri and İversen 1964, Aytuğ 1967, Moore et al. 1991). The pollen types present in the honey samples were counted and classified according to their percentages, as dominant pollen (more than $45 \%$ of the pollen grains counted), secondary pollen $(16 \%-44 \%)$, minor pollen (3\% - 15\%), and rare pollen (less than 3\%) (Louveaux 1970).

\section{Results and Discussion}

Seven samples produced in the vicinity of Yı̆̆llca Balköy Honey Production Forest were analyzed in this study (Table 1). Fourth-three pollen types from 26 families were recognized in the produced in the during honey harvest periods from May to August, and definitions of dominant, secondary, minor and rare pollen spectrum in honey samples were determined (Table 2).

Table 2: Taxa detected in honey samples and definitions of dominant (D), secondary (S), minor (M) and rare (R) pollen spectrum.

\begin{tabular}{|c|c|c|c|c|c|c|c|c|}
\hline \multirow[t]{2}{*}{ Families } & \multirow[t]{2}{*}{ Taxa } & \multicolumn{7}{|c|}{ Honey Samples and Pollen Spectra } \\
\hline & & Y01 & Y02 & Y03 & Y04 & Y05 & Y06 & Y07 \\
\hline Adoxaceae & Sambucus ebulus L. & & & & $\mathrm{R}$ & & $\mathrm{R}$ & $\mathrm{R}$ \\
\hline Apiaceae & & $\mathrm{R}$ & $\mathrm{R}$ & & $\mathrm{R}$ & $\mathrm{R}$ & $\mathrm{R}$ & $\mathrm{R}$ \\
\hline \multirow{4}{*}{ Asteraceae } & Arctium minus (Hill) Bernh. & & $\mathrm{R}$ & & $\mathrm{R}$ & & $\mathrm{R}$ & $\mathrm{R}$ \\
\hline & Centaurea L. sp. & $\mathrm{R}$ & $\mathrm{R}$ & $\mathrm{R}$ & $\mathrm{R}$ & & $\mathrm{R}$ & \\
\hline & Cirsium Mill. sp. & $\mathrm{R}$ & & $\mathrm{R}$ & $\mathrm{R}$ & & & \\
\hline & Cota tinctoria var. pallida (DC.) Özbek \& Vural & & & & $\mathrm{R}$ & & & \\
\hline Betulaceae & Alnus glutinosa (L.) Geartner subsp. glutinosa & & & & $\mathrm{R}$ & $\mathrm{R}$ & & \\
\hline Boraginaceae & Echium vulgare L. & & & & & & $\mathrm{R}$ & \\
\hline Brassicaceae & Rorippa sylvestris (L.) Besser & $\mathrm{R}$ & $\mathrm{R}$ & $\mathrm{R}$ & $\mathrm{R}$ & & $\mathrm{R}$ & $\mathrm{R}$ \\
\hline Campanulaceae & Campanula L. sp. & & & $\mathrm{R}$ & & & & $\mathrm{R}$ \\
\hline Cupressaceae & Cupressus L. sp. & & & $\mathrm{R}$ & & $\mathrm{R}$ & $\mathrm{R}$ & \\
\hline Ericaceae & Rhododendron ponticum L. & $\mathrm{D}$ & $\mathrm{D}$ & $\mathrm{D}$ & $\mathrm{S}$ & $\mathrm{R}$ & $\mathrm{D}$ & $\mathrm{R}$ \\
\hline Euphorbiaceae & Euphorbia L. sp. & & & & & & $\mathrm{R}$ & \\
\hline \multirow{6}{*}{ Fabaceae } & Lathyrus laxiflorus (Desf.) Kuntze subsp. laxiflorus & & $\mathrm{R}$ & & $\mathrm{R}$ & $\mathrm{R}$ & & $\mathrm{R}$ \\
\hline & Trifolium L. sp. & $\mathrm{R}$ & $\mathrm{R}$ & $\mathrm{R}$ & $\mathrm{R}$ & & $\mathrm{R}$ & $\mathrm{R}$ \\
\hline & Lotus corniculatus L. var. tenuifolius & $\mathrm{R}$ & & & $\mathrm{R}$ & & $\mathrm{R}$ & $\mathrm{R}$ \\
\hline & Dorycnium graecum (L.) Ser. & $\mathrm{R}$ & $\mathrm{R}$ & & & & $\mathrm{R}$ & \\
\hline & Medicago lupulina $\mathrm{L}$. & & & & & & $\mathrm{R}$ & \\
\hline & Galega officinalis L. & & & & & & $\mathrm{R}$ & \\
\hline \multirow{3}{*}{ Fagaceae } & Castanea sativa Mill. & & & & & $\mathrm{D}$ & $\mathrm{M}$ & $\mathrm{D}$ \\
\hline & Fagus orientalis Lipsky & $\mathrm{S}$ & $\mathrm{S}$ & $\mathrm{S}$ & $\mathrm{S}$ & $\mathrm{R}$ & $\mathrm{R}$ & $\mathrm{R}$ \\
\hline & Quercus L. sp. & $\mathrm{M}$ & $\mathrm{M}$ & $\mathrm{M}$ & $\mathrm{M}$ & $\mathrm{R}$ & $\mathrm{M}$ & $\mathrm{M}$ \\
\hline Gentianaceae & Centaurium erythraea Rafn subsp. erythraea & & & & & & & $\mathrm{R}$ \\
\hline Geraniaceae & Geranium L. sp. & $\mathrm{R}$ & $\mathrm{R}$ & $\mathrm{R}$ & $\mathrm{R}$ & $\mathrm{R}$ & $\mathrm{R}$ & $\mathrm{R}$ \\
\hline Iridaceae & Iris sintenisii Janka & $\mathrm{R}$ & & & & & $\mathrm{R}$ & \\
\hline \multirow{3}{*}{ Lamiaceae } & Mentha L. sp. & & $\mathrm{R}$ & $\mathrm{R}$ & & & & \\
\hline & Salvia L. sp. & & & & $\mathrm{R}$ & & & $\mathrm{R}$ \\
\hline & Stachys L. sp. & $\mathrm{R}$ & & & & & & \\
\hline Oleacea & Ligustrum vulgare L. & & & $\mathrm{R}$ & $\mathrm{R}$ & & $\mathrm{R}$ & $\mathrm{R}$ \\
\hline \multirow{2}{*}{ Onagraceae } & Circaea lutetiana L. & $\mathrm{R}$ & $\mathrm{R}$ & & $\mathrm{R}$ & & & \\
\hline & Epilobium L. sp. & & & & & & $\mathrm{R}$ & \\
\hline Pinaceae & Pinus sylvestris L. var. hamata Steven & & & & $\mathrm{R}$ & $\mathrm{R}$ & $\mathrm{R}$ & \\
\hline Plantaginaceae & Plantago L. sp. & $\mathrm{R}$ & $\mathrm{R}$ & & & $\mathrm{R}$ & $\mathrm{R}$ & $\mathrm{R}$ \\
\hline Poaceae & Holcus lanatus L. & & & & $\mathrm{R}$ & & & \\
\hline Primulaceae & Anagallis arvensis var. arvensis L. & & & & $\mathrm{R}$ & & & \\
\hline Ranunculaceae & Ranunculus neapolitanus Ten. & $\mathrm{R}$ & $\mathrm{R}$ & & $\mathrm{R}$ & & & \\
\hline \multirow{6}{*}{ Rosaceae } & Agrimonia repens $\mathrm{L}$. & $\mathrm{R}$ & $\mathrm{R}$ & $\mathrm{R}$ & $\mathrm{R}$ & & $\mathrm{R}$ & $\mathrm{R}$ \\
\hline & Pyracantha coccinea M.Roem. & $\mathrm{R}$ & $\mathrm{R}$ & $\mathrm{R}$ & $\mathrm{R}$ & $\mathrm{R}$ & & $\mathrm{R}$ \\
\hline & Crataegus L. sp. & $\mathrm{R}$ & $\mathrm{R}$ & $\mathrm{R}$ & $\mathrm{R}$ & $\mathrm{R}$ & $\mathrm{R}$ & $\mathrm{R}$ \\
\hline & Rubus L. sp. & $\mathrm{R}$ & $\mathrm{R}$ & & $\mathrm{R}$ & $\mathrm{R}$ & $\mathrm{M}$ & $\mathrm{M}$ \\
\hline & Rosa canina $\mathrm{L}$. & $\mathrm{R}$ & & & & & $\mathrm{M}$ & $\mathrm{R}$ \\
\hline & Potentilla L. sp. & & & & & & $\mathrm{R}$ & $\mathrm{R}$ \\
\hline Scrophulariaceae & Verbascum blattaria $\mathrm{L}$. & & & & $\mathrm{R}$ & & & \\
\hline Urticaceae & Urtica dioica $\mathrm{L}$ & & & & & & $\mathrm{R}$ & \\
\hline
\end{tabular}

Rhododendron ponticum L. was dominant with the pollen percentage of 50.4 in Y01 (Table 3; Figure

2). Fagus orientalis Lipsky with the pollen percentage of 28.6 was determined as secondary and Quercus 
L. sp. with the pollen percentage of 10.6 was determined as minor. As a trace in Y01, Agrimonia repens L., Apiaceae, Centaurea L. sp., Circaea lutetiana L., Cirsium Mill. sp., Crataegus L. sp., Dorycnium graecum (L.) Ser., Geranium L. sp., Iris sintenisii Janka, Lotus corniculatus L. var. tenuifolius, Plantago L. sp., Pyracantha coccinea M.Roem., Ranunculus neapolitanus Ten., Rorippa sylvestris (L.) Besser, Rosa canina L., Rubus L. sp., Stachys L. sp. and Trifolium L. sp. were identified (Table 3; Figure 2).

In the honey sample of Y02, Rhododendron ponticum L. was dominant with the pollen percentage of 48.2 and Fagus orientalis Lipsky was secondary with the percentage of 35 (Table 3; Figure 2). Quercus L. sp. with the pollen percentage of 6.4 was identified as minor. Trace pollen grains belong to Agrimonia repens L., Apiaceae, Arctium minus (Hill) Bernh., Centaurea L. sp., Circaea lutetiana L., Crataegus L. sp., Dorycnium graecum (L.) Ser., Geranium L. sp., Lathyrus laxiflorus (Desf.) Kuntze subsp. laxiflorus, Mentha L. sp., Plantago L. sp., Pyracantha coccinea M.Roem., Ranunculus neapolitanus Ten., Rorippa sylvestris (L.) Besser, Rubus sp. and Trifolium sp. in Y02 (Table 3; Figure 2).

Rhododendron ponticum L. was dominant with the pollen percentage of 47.3 in Y03 (Table 3; Figure 2). Fagus orientalis Lipsky with the pollen percentage of 27.3 was determined as secondary and Quercus L. sp. with the pollen percentage of 10.6 was determined as minor. As a rare in Y03, Agrimonia repens L., Campanula L. sp., Centaurea L. sp., Cirsium Mill. sp., Crataegus L. sp., Cupressus L. sp., Geranium L. sp., Ligustrum vulgare L., Mentha L. sp., Pyracantha coccinea M.Roem., Rorippa sylvestris (L.) Besser and Trifolium L. sp. were identified (Table 3; Figure 2).

In the honey sample of Y04; Fagus orientalis Lipsky and Rhododendron ponticum L. were secondary with the percentage of 34.1 and 29 respectively (Table 3; Figure 2). Quercus sp. with the pollen percentage of 15.2 was identified as minor. Trace pollen grains belong to Agrimonia repens L., Alnus glutinosa (L.) Geartner subsp. glutinosa, Anagallis arvensis var. arvensis L., Apiaceae, Arctium minus (Hill) Bernh., Centaurea L. sp., Circaea lutetiana L., Cirsium Mill. sp., Cota tinctoria var. pallida (DC.) Özbek \& Vural, Crataegus L. sp., Geranium L. sp., Holcus lanatus L., Lathyrus laxiflorus (Desf.) Kuntze subsp. laxiflorus, Ligustrum vulgare L., Lotus corniculatus L. var. tenuifolius, Pinus sylvestris L. var. hamata Steven, Pyracantha coccinea M.Roem., Ranunculus neapolitanus Ten., Rorippa sylvestris (L.) Besser, Rubus L. sp., Salvia L. sp., Sambucus ebulus L., Trifolium L. sp. and Verbascum blattaria L. in Y04 (Table 3; Figure 2).

Castanea sativa Mill. was dominant with the pollen percentage of 91.5 in Y05 (Table 3; Figure 2). As a trace, Alnus glutinosa (L.) Geartner subsp. glutinosa, Apiaceae, Crataegus L. sp., Cupressus L. sp., Fagus orientalis Lipsky, Geranium L. sp., Lathyrus laxiflorus (Desf.) Kuntze subsp. laxiflorus, Pinus sylvestris L. var. hamata Steven, Plantago L. sp., Pyracantha coccinea M.Roem., Quercus L. sp., Rhododendron ponticum L. and Rubus L. sp. were determined in Y05 (Table 3; Figure 2).

Rhododendron ponticum L. was dominant with the pollen percentage of 58.9 in Y06 (Table 3; Figure 2). Rubus sp. (\%11.3), Castanea sativa Mill. (\%4.9), Rosa canina L. (\%4.6) and Quercus L. sp. (\%4) were identified as minor. Rare pollen grains belong to Agrimonia repens L., Apiaceae, Arctium minus (Hill) Bernh., Centaurea L. sp., Crataegus L. sp., Cupressus L. sp., Dorycnium graecum (L.) Ser., Echium vulgare L., Epilobium L. sp., Euphorbia L. sp., Fagus orientalis Lipsky, Galega officinalis L., Geranium L. sp., Iris sintenisii Janka, Ligustrum vulgare L., Lotus corniculatus L. var. tenuifolius, Medicago lupulina L., Pinus sylvestris L. var. hamata Steven, Plantago L. sp., Rorippa sylvestris (L.) Besser, Sambucus ebulus L., Trifolium L. sp. and Urtica dioica L. in Y06 (Table 3; Figure 2). 
Castanea sativa Mill. was dominant with the pollen percentage of 92.5 in Y07 (Table 3; Figure 2). Quercus L. sp. with the pollen percentage of 8.8 and Rubus L. sp. with the pollen percentage of 3.1 were identified as minor. As a rare in Y07, Agrimonia repens L., Apiaceae, Arctium minus (Hill) Bernh., Campanula L. sp., Centaurium erythraea Rafn subsp. erythraea, Crataegus L. sp., Fagus orientalis Lipsky, Geranium L. sp., Lathyrus laxiflorus (Desf.) Kuntze subsp. laxiflorus, Ligustrum vulgare L., Lotus corniculatus L. var. tenuifolius, Plantago L. sp., Potentilla L. sp., Pyracantha coccinea M.Roem., Rhododendron ponticum L., Rorippa sylvestris (L.) Besser, Rosa canina L., Salvia L. sp. and Sambucus ebulus L. were determined (Table 3; Figure 2).

Table 3: Pollen types in the honey samples (in percentages).

\begin{tabular}{|c|c|c|c|c|}
\hline $\begin{array}{l}\text { Sample } \\
\text { number }\end{array}$ & $\begin{array}{l}\text { Dominant pollen } \\
(>=45 \%)\end{array}$ & $\begin{array}{l}\text { Secondary pollen } \\
(16-44 \%)\end{array}$ & $\begin{array}{l}\text { Minor pollen } \\
(3-15 \%)\end{array}$ & $\begin{array}{l}\text { Rare pollen } \\
(<3 \%)\end{array}$ \\
\hline Y01 & $\begin{array}{l}\text { Rhododendron ponticum } \\
\text { L. } \% 50.4\end{array}$ & $\begin{array}{l}\text { Fagus orientalis Lipsky } \\
\% 28.6\end{array}$ & $\begin{array}{l}\text { Quercus L. sp. } \\
\% 10.6\end{array}$ & $\begin{array}{l}\text { Agrimonia repens L., Apiaceae, Centaurea L. sp., Circaea lutetiana L., } \\
\text { Cirsium Mill. sp., Crataegus L. sp., Dorycnium graecum (L.) Ser., } \\
\text { Geranium L. sp., Iris sintenisii Janka, Lotus corniculatus L. var. } \\
\text { tenuifolius, Plantago L. sp., Pyracantha coccinea M.Roem., } \\
\text { Ranunculus neapolitanus Ten., Rorippa sylvestris }(\mathrm{L} .) \text { Besser, Rosa } \\
\text { canina L., Rubus L. sp., Stachys L. sp., Trifolium L. sp. }\end{array}$ \\
\hline Y02 & $\begin{array}{l}\text { Rhododendron ponticum } \\
\text { L. } \% 48.2\end{array}$ & $\begin{array}{l}\text { Fagus orientalis Lipsky } \\
\% 35\end{array}$ & $\begin{array}{l}\text { Quercus L. sp. } \\
\% 6.4\end{array}$ & $\begin{array}{l}\text { Agrimonia repens L., Apiaceae, Arctium minus (Hill) Bernh., } \\
\text { Centaurea } \text { L. sp., Circaea lutetiana L., Crataegus L. sp., Dorycnium } \\
\text { graecum } \text { (L.) Ser., Geranium L. sp., Lathyrus laxiflorus (Desf.) Kuntze } \\
\text { subsp. laxiflorus, Mentha L. sp. Plantago L. sp., Pyracantha coccinea } \\
\text { M.Roem., Ranunculus neapolitanus Ten., Rorippa sylvestris (L.) } \\
\text { Besser, Rubus L. sp., Trifolium L. sp. }\end{array}$ \\
\hline Y03 & $\begin{array}{l}\text { Rhododendron ponticum } \\
\text { L. \%47.3 }\end{array}$ & $\begin{array}{l}\text { Fagus orientalis Lipsky } \\
\% 27.3\end{array}$ & $\begin{array}{l}\text { Quercus sp. } \\
\% 10.6\end{array}$ & $\begin{array}{l}\text { Agrimonia repens } \text { L., Campanula } \text { L. sp., Centaurea } \text { L. sp., Cirsium } \\
\text { Mill. sp., Crataegus L. sp., Cupressus L. sp., Geranium L. sp., } \\
\text { Ligustrum vulgare L., Mentha L. sp., Pyracantha coccinea M.Roem., } \\
\text { Rorippa sylvestris }(\text { L.) Besser, Trifolium L. sp. }\end{array}$ \\
\hline Y04 & & $\begin{array}{l}\text { Fagus orientalis Lipsky } \\
\% 34.1, \text { Rhododendron } \\
\text { ponticum L. } \% 29\end{array}$ & $\begin{array}{l}\text { Quercus L. sp. } \\
\% 15.2\end{array}$ & $\begin{array}{l}\text { Agrimonia repens L., Alnus glutinosa (L.) Geartner subsp. glutinosa, } \\
\text { Anagallis arvensis var. arvensis L., Apiaceae, Arctium minus (Hill) } \\
\text { Bernh., Centaurea L. sp., Circaea lutetiana L., Cirsium Mill. sp., Cota } \\
\text { tinctoria var. pallida (DC.) Özbek \& Vural, Crataegus L. sp., } \\
\text { Geranium L. sp., Holcus lanatus L. Lathyrus laxiflorus (Desf.) Kuntze } \\
\text { subsp. laxiflorus, Ligustrum vulgare L., Lotus corniculatus L. var. } \\
\text { tenuifolius, Pinus sylvestris L. var. hamata Steven, Pyracantha } \\
\text { coccinea M.Roem., Ranunculus neapolitanus Ten., Rorippa sylvestris } \\
\text { (L.) Besser, Rubus L. sp., Salvia L. sp., Sambucus ebulus L., Trifolium } \\
\text { L. sp., Verbascum blattaria L. }\end{array}$ \\
\hline Y05 & $\begin{array}{l}\text { Castanea sativa Mill. } \\
\% 91.5\end{array}$ & & & $\begin{array}{l}\text { Alnus glutinosa (L.) Geartner subsp. glutinosa, Apiaceae, Crataegus L. } \\
\text { sp., Cupressus L. sp., Fagus orientalis Lipky, Geranium L. sp., } \\
\text { Lathyrus laxiflorus (Desf.) Kuntze subsp. laxiflorus, Pinus sylvestris L. } \\
\text { var. hamata Steven, Plantago L. sp., Pyracantha coccinea M.Roem., } \\
\text { Quercus L. sp., Rhododendron ponticum L., Rubus L. sp. }\end{array}$ \\
\hline Y06 & $\begin{array}{l}\text { Rhododendron ponticum } \\
\text { L. } \% 58.9\end{array}$ & & $\begin{array}{l}\text { Castanea sativa } \\
\text { Mill. \% } 4.9, \text { Rosa } \\
\text { canina } \text { L. \% } 4.6 \text {, } \\
\text { Quercus L. sp. } \\
\% 4, \text { Rubus } \text { L. sp. } \\
\% 11.3\end{array}$ & $\begin{array}{l}\text { Agrimonia repens L., Apiaceae, Arctium minus (Hill) Bernh., } \\
\text { Centaurea } \text { L. sp., Crataegus L. sp., Cupressus L. sp., Dorycnium } \\
\text { graecum (L.) Ser., Echium vulgare L., Epilobium L. sp., Euphorbia } \mathrm{L} \text {. } \\
\text { sp., Fagus orientalis Lipsky, Galega officinalis L., Geranium L. sp., } \\
\text { Iris sintenisii Janka, Ligustrum vulgare L., Lotus corniculatus L. var. } \\
\text { tenuifolius, Medicago lupulina L., Pinus sylvestris L. var. hamata } \\
\text { Steven, Plantago L. sp., Potentilla L. sp., Rorippa sylvestris (L.) } \\
\text { Besser, Sambucus ebulus L., Trifolium L. sp., Urtica dioica L. }\end{array}$ \\
\hline Y07 & $\begin{array}{l}\text { Castanea sativa Mill. } \\
\% 92.5\end{array}$ & & $\begin{array}{l}\text { Quercus L. sp. } \\
\% 8.8, \text { Rubus L. } \\
\text { sp. } \% 3.1\end{array}$ & $\begin{array}{l}\text { Agrimonia repens L., Apiaceae, Arctium minus (Hill) Bernh., } \\
\text { Campanula L. sp., Centaurium erythraea Rafn subsp. erythraea, } \\
\text { Crataegus L. sp., Fagus orientalis Lipsky, Geranium L. sp., Lathyrus } \\
\text { laxiflorus (Desf.) Kuntze subsp. laxiflorus, Ligustrum vulgare L. } \\
\text { Lotus corniculatus L. var. tenuifolius, Plantago L. sp., Potentilla L. sp., } \\
\text { Pyracantha coccinea M.Roem., Rhododendron ponticum L., Rorippa } \\
\text { sylvestris (L.) Besser, Rosa canina L., Salvia L. sp., Sambucus ebulus } \\
\text { L., Trifolium L. sp. }\end{array}$ \\
\hline
\end{tabular}



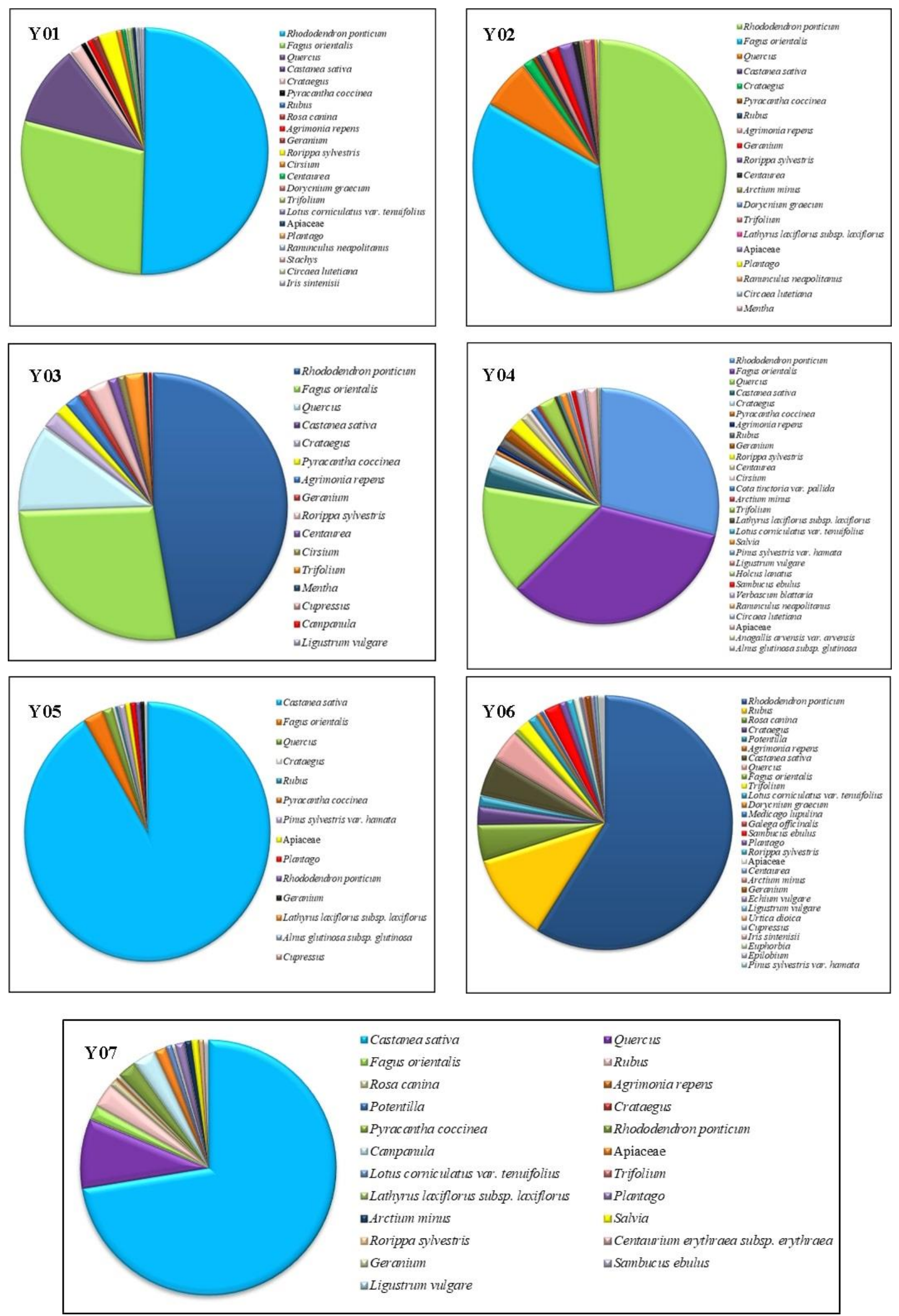

Figure 2: Percentage diagrams of dominant, secondary, minor and trace pollens in honey samples. 
As a result of pollen analysis performed on 7 honey samples taken from the vicinity of Yiğılca Balköy Honey Production Forest, the pollen list was obtained by taking images of the pollen species detected in the honey samples by using a Light Microscope (Figure 3-5).
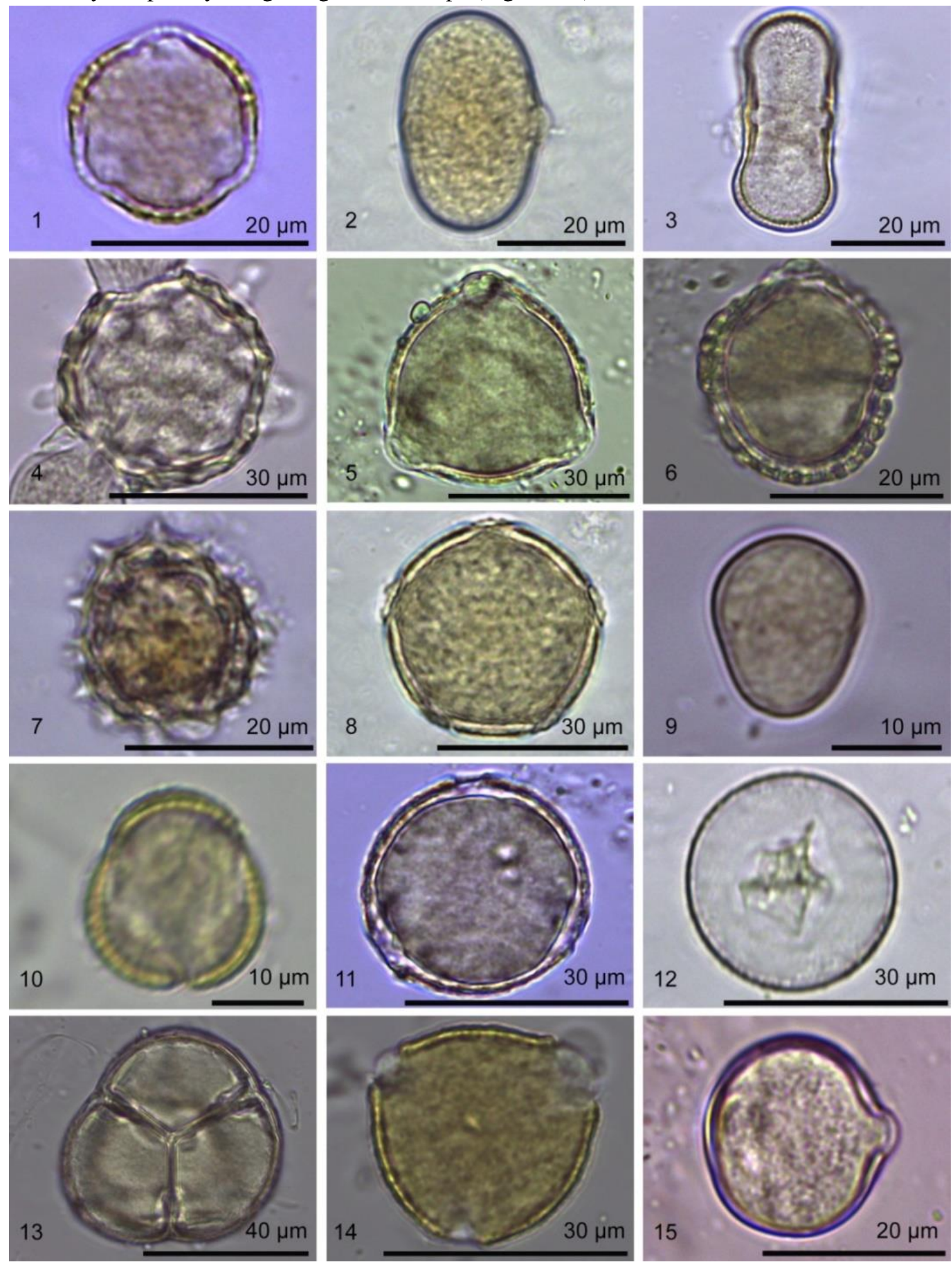

Figure 3: Light microscope photographs of taxa identified in honey samples. Adoxaceae: 1, Sambucus ebulus L.; Apiaceae: 2-3; Asteraceae: 4, Arctium minus (Hill) Bernh.; 5, Centaurea L. sp.; 6, Cirsium L. sp.; 7, Cota tinctoria var. pallida (DC.) Özbek \& Vural; Betulaceae: 8, Alnus glutinosa subsp. glutinosa; Boraginaceae: 9, Echium vulgare L.; Brassicaceae: 10, Rorippa sylvestris (L.) Besser; Campanulaceae: 11, Campanula L. sp.; Cupressaceae: 12, Cupressus L. sp.; Ericaceae: 13, Rhododendron ponticum L.; Euphorbiaceae: 14, Euphorbia L. sp.; Fabaceae: 15, Dorycnium graecum (L.) Ser. 

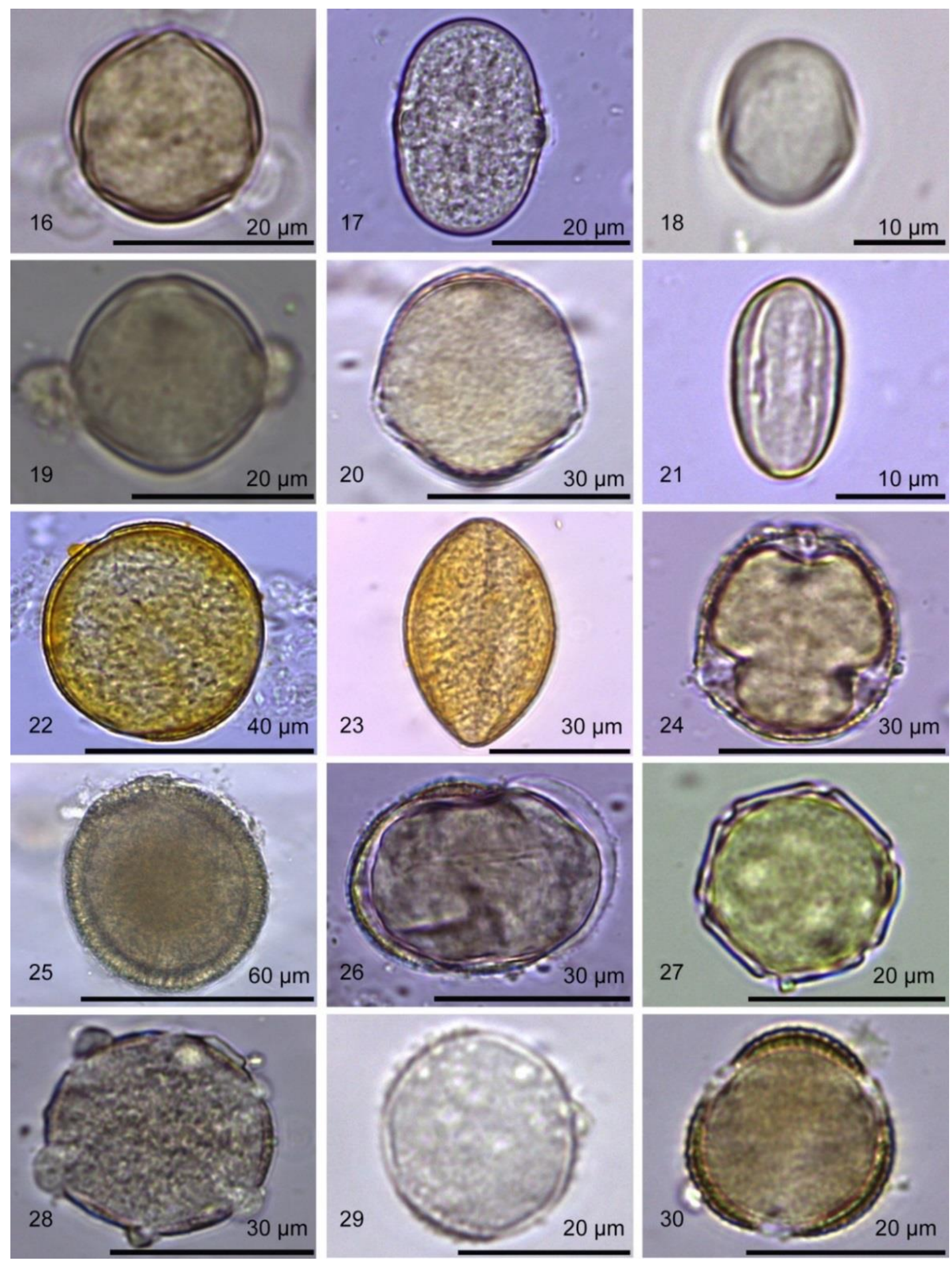

Figure 4: Light microscope photographs of taxa identified in honey samples. Fabaceae: 16, Galega officinalis L.; 17, Lathyrus laxiflorus (Desf.) Kuntze subsp. laxiflorus; 18, Lotus corniculatus L. var. tenuifolius L.; 19, Medicago lupulina L.; 20, Trifolium L. sp.; Fagaceae: 21, Castanea sativa Mill.; 22, Fagus orientalis Lipsky; 23-24, Quercus L. sp.; Gentianaceae: 81, Centaurium erythraea Rafn subsp. erythraea; Geraniaceae: 25, Geranium L. sp.; Iridaceae: 26, Iris sintenisii Janka; Lamiaceae: 27, Mentha L. sp.; 28, Salvia L. sp.; 29, Stachys L. sp.; Oleaceae: 30, Ligustrum vulgare $\mathrm{L}$. 

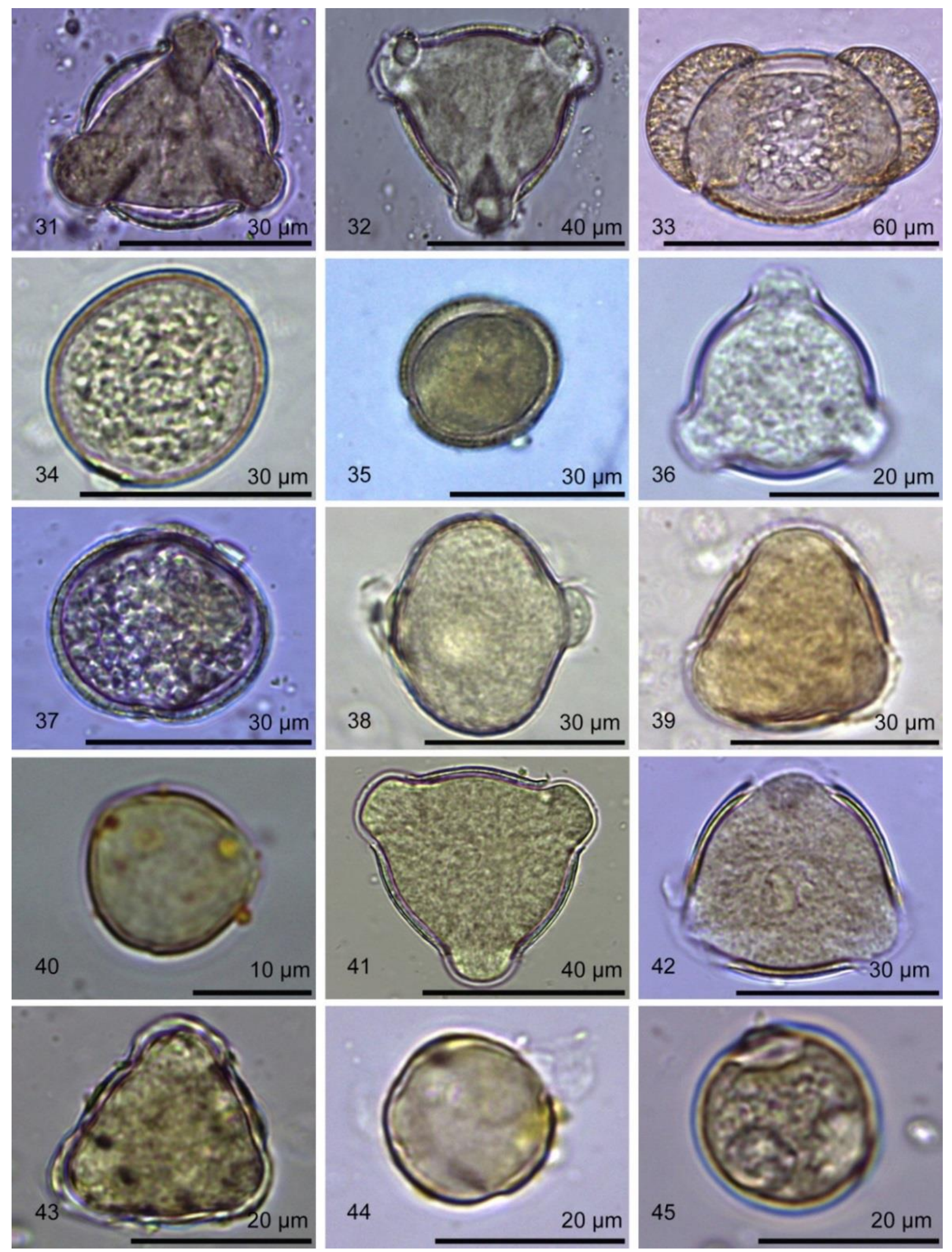

Figure 5: Light microscope photographs of taxa identified in honey samples. 31, Onagraceae: Circaea lutetiana L.; 32, Epilobium L. sp.; 33, Pinaceae: Pinus sylvestris L. var. hamata Steven; Plantaginaceae: 34, Plantago L. sp.; Poaceae: 35, Holcus lanatus L.; Primulaceae: 36, Anagallis arvensis var. arvensis L.; Ranunculaceae: 37, Ranunculus neapolitanus Ten.; Rosaceae: 38, Agrimonia repens L.; 39, Crataegus L. sp.; 40, Potentilla L. sp.; 41, Pyracantha coccinea M.Roem.; 42, Rosa canina L.; 43, Rubus L. sp.; Scrophulariaceae: 44, Verbascum blattaria L.; Urticaceae: 45, Urtica dioica $\mathrm{L}$. 


\section{Conclusion}

Pollen analysis was carried out on 7 honey samples produced in the vicinity of Yığılca Balköy Honey Production Forest. Pollen grains of Rhododendron ponticum L., Fagus orientalis Lipsky, Castanea sativa Mill., Agrimonia repens L., Pyracantha coccinea M.Roem., Rosa canina L., Sambucus ebulus L., Arctium minus (Hill) Bernh., Cota tinctoria var. pallida (DC.) Özbek \& Vural, Alnus glutinosa (L.) Geartner subsp. glutinosa, Echium vulgare L., Rorippa sylvestris (L.) Besser, Dorycnium graecum (L.) Ser., Galega officinalis L., Lathyrus laxiflorus (Desf.) Kuntze subsp. laxiflorus, Lotus corniculatus L. var. tenuifolius, Medicago lupulina L., Centaurium erythraea Rafn subsp. erythraea, Iris sintenisii Janka, Ligustrum vulgare L., Circaea lutetiana L., Pinus sylvestris L. var. hamata Steven, Holcus lanatus L., Anagallis arvensis var. arvensis L., Ranunculus neapolitanus Ten., Verbascum blattaria L. and Urtica dioica L. were identified on the basis of 27 taxa. In addition, 15 pollen types belong to genus level were detected in all honey samples, including Quercus L., Crataegus L., Potentilla L., Rubus L., Centaurea L., Cirsium Mill., Campanula L., Cupressus L., Euphorbia L., Trifolium L., Geranium L., Mentha L., Salvia L., Stachys L. and Plantago L. Quercus pollens identified in all honey samples belong to the "Quercus cerris group" (Deciduous Quercus Group)".

Of the 7 honey samples, Rhododendon ponticum L. pollen was determined $50.4 \%$ in Y01; $48.2 \%$ in Y02; 47.3\% in Y03 and 58.9\% in Y06. The pollen percentage of Rhododendon ponticum L. is higher than $45 \%$, so these honeys can be named as "rhododendron honey". Honey samples of Y01, Y02 and Y06 correspond to the names taken from the manufacturers. However, no pollen percentage is specified for Rhododendron honey in the Turkish Food Codex-Honey Notification (TOB 2020). The minimum amount of Rhododendron pollen percentage data should be added to the honey statement in order for the rhododendron honey to be fully identified.

Fagaceae family (Fagus orientalis Lipsky 34.1\%; Quercus L. sp. 15.2\%) was detected with the pollen percentage of 49.3 in Y04. This honey sample taken as flower honey from the manufacturer, after this analysis it can be called Fagaceae family honey.

Pollen grains of Castanea sativa Mill. were found as dominant at a rate of $91.5 \%$ in Y05 and $92.5 \%$ in Y07. As stated in the Turkish Food Codex Honey Communiqué, Castanea sativa Mill. pollens must be above $70 \%$ of the values obtained as a result of pollen analysis on honey samples (TOB 2020). Therefore, since the pollen percentage of Castanea sativa Mill. was detected above $70 \%$ in these honey samples, these two honeys (Y05 and Y07) should be called "Castanea honey" instead of "flower honey".

In a study, pollen analyzes were also carried out in honey samples taken from Bolu province, which is close to the Yığılca region. As a result, Rhododendron pollen grains were identified as dominant and Erica pollen grains were secondary (Kaya et. al. 2005). It is seen that these results are similar to the presented study. In another study, pollen analyzes were made in chestnut and mad honey samples taken from Akçakoca and Yığılca districts. As a result of this analysis, Castanea sativa Mill. pollen was determined as dominant in chestnut and mad honey samples and these samples were classified as monofloral honey. In the mad honey samples, the important minor pollens were determined as Rhododendron ponticum L. and Lysimachia verticillaris Spreng., and they were named as multifloral honey because they also contained the pollen grains of the other five species (Gürdal and Sönmez 2021).

Plant species were determined in the Yığllca region according to the pollen analysis performed on honey samples. The most preferred plant species by Yiğılca bee were identified as Rhododendron ponticum L., Castanea sativa Mill. and Fagus orientalis Lipsky, which were found as dominant and secondary in 
all honey samples. Six samples of honey were unifloral ( $>45 \%$ pollen of one taxon in sample) with four being unifloral for Rhododendron ponticum L. and two for Castanea sativa Mill. One sample of honey was classified as multifloral with the percentages of secondary and rare pollen groups. In addition, the determination of the presence of pollen grains belonging to the melliferous plants of the region contributed to the current and prospective flora studies and also created a pollen database for the future pollen atlas studies of Düzce region.

Acknowledgments: This article was produced from the master thesis titled "Pollen Analysis of Yigilca Region Honey and Melliferous Plants" and supported by the Scientific Research Projects Coordination Unit of Duzce University under Grant [project number BAP-2019.02.02.1031].

\section{References}

Altay, V., Karahan, P., Karahan, F., Öztürk, M. (2018). Pollen analysis of honeys from Hatay/Turkey. Biological Diversity and Conservation, 11(3), 209-222.

Aytuğ, B. (1967). Polen Morfolojisi ve Türkiye'nin Önemli Gymnospermleri Üzerinde Palinolojik Araştırmalar. İstanbul Üniversitesi Orman Fakültesi Yayınları, İstanbul.

Davis, P.H., (1965). Flora of Turkey and thu East Aegean Islands. Edinburgh: Edinburgh University Press.

Doğan, C., Sorkun, K. (2001). Pollen analysis of honeys from Aegean, Marmara, Mediterranean and Black Sea Regions in Turkey. Mellifera, 1(1), 34-44.

Erdoğan, N., Pehlivan, S., Doğan, C. (2006). Pollen analysis of honeys from Hendek, Akyazı and Kocaali districts of Adapazarı province (Turkey). Mellifera, 6(10-12), 20-27.

Erdtman, G. (1952). Pollen morphology and plant taxonomy-angiosperms. The Chronica Botanica Company, Waltham, US.

Erdtman, G. (1957). Pollen and spore morphology/plant taxonomy Gymnospermae, Pteridopyhta, Bryophyta. Almqvist \& Wiksell, Stockholm, SE.

Faegri, K; Iversen, J. (1964). Textbook of pollen analysis. John Wiley and Sons, Munksgaard, DK.

Gösterit, A., Kekeçoğlu, M., Çıkılı, Y. (2012). Yığılca yerel bal arısının bazı performans özellikleri bakımından Kafkas ve Anadolu bal arısı ırkı melezleri ile karşılaştırılması. Süleyman Demirel Üniversitesi Ziraat Fakültesi Dergisi 7(1), 107-114.

Gürdal, B., Sönmez, S. (2021). Pollen and physicochemical analysis of honey samples from Akçakoca and Yığılca district (Western Black Sea). Yuzuncu Yil University Journal of Agricultural Sciences, 31(3), 576-586.

http://cografyaharita.com/haritalarim/41_duzce_ili_haritasi.png (Visited on date: 14/12/2021).

Kambur, M., Kekeçoğlu, M., Yıldız, İ. (2015). Düzce ili Yığıllca ilçesinde üretilen balların kimyasal ve palinolojik analiz yöntemleri ile değerlendirilmesi. Uludağ Arıcılık Dergisi, 15(2), 67-79.

Kaya, Z., Binzet, R., Orcan, N. (2005). Pollen analysis of honeys from some regions in Turkey. Apiacta, 40, 1015.

Kekeçoğlu, M. (2007). 'Türkiye Bal Arılarının mtDNA ve Bazı Morfolojik Özellikleri Bakımından Karşılaştııılmasına Yönelik Bir Araştırma', Doktora Tezi, Namık Kemal Üniversitesi Fen Bilimleri Enstitüsü, Tekirda ğ, Türkiye.

Kekeçoğlu, M. (2010). Honey bee biodiversity in Western Black Sea and evidence for a new honey bee ecotype in Yığılca provinces of Düzce city. Biyoloji Bilimleri Araştırma Dergisi, 3(1), 73-78. 
Louveaux, J. (1970). Annexes microphotographiques aux méthodes officielles d'analyse. Tome III, Atlas photographique d'analyse pollinique des miels, Service de la répression des fraudes et du contrôle de la qualité, Paris.

Moore, P., Webb, J.A., Collinson, M.E. (1991). Pollen Analysis, 2nd Edition, Blackwell, Oxford, 216 pp.

Özler, H. (2015). Melissopalynological analysis of honey samples belonging to different districts of Sinop, Turkey. Mellifera, 15(1), 1-11.

Özler, H. (2018). Güney Anadolu Bölgesine ait ballarda polen analizi. Uludağ Arıcılık Dergisi, 18(2), 73-86.

Sawyer, R. (1981). Pollen Identification for Beekeepers. University College Cardiff Press, Cardiff, US.

Silici, S., Gökçeoğlu, M. (2007). Pollen analysis of honeys from Mediterranean Region of Anatolia. Grana, 46(1), 57-64.

Sorkun, K. (2008). Türkiye’nin Nektarlı Bitkileri, Polenleri ve Balları. Palme Yayıncılık, Ankara, TR.

Sorkun, K., Doğan, C. (1995). Pollen analysis in honey collected from different regions of Turkey. Hacettepe Bulletin of Natural Sciences and Engineering, 16, 15-24.

Sorkun, K., İnceoğlu, Ö. (1984). Dominant pollens in honeys of Central Anatolia Region. Nature Science Journal, $8(3), 377-381$.

Şık, L., Güvensen, A., Durmuşkahya, C., Erol, O. (2017). Pollen analysis of honeys from Ardahan/Turkey. Biological Diversity and Conservation, 10(2), 12-19.

Tarım ve Orman Bakanlığı (TOB) (2020). Türk Gıda Kodeksi-Bal Tebliği. https://www.resmigazete.gov.tr/eskiler/2020/04/20200422-13.htm/ (Visited on date: 03/11/2020).

Woodhouse, R. P. (1935). Pollen Grains. Hafner Publishing Company, New York, NY.

Yıldırım, E.A., Güneş Özkan, N., Karlığ̆lı Kılıç, N. (2020). Yığılca (Düzce) Balköy honey forest flora. Düzce University Journal of Forestry, 16(2), 45-69.

Yılmaz, A., Işık, Ö., Yıldırım, S. (2017). Yığılca Balköy Bal Üretim Ormanı Uygulama Projesi. Orman Genel Müdürlüğü, Düzce, TR. 